\title{
Otimização e validação do método analítico volumétrico para quantificação do ácido tioglicólico em cremes cosméticos
}

\author{
Optimization and validation of the volumetric analytical method for \\ the quantification of thioglycolic acid in cosmetic creams
}

Recebido em: 19/12/2016

Aceito em: $\quad 03 / 07 / 2017$

\author{
Maria Cristina SANTA BARBARA; Ligia Luriko MIYAMARU \\ Instituto Adolfo Lutz. Centro de Medicamentos, Cosméticos e Saneantes. Núcleo de \\ Ensaios Físicos e Químicos em Cosméticos e Saneantes. Av. Dr Arnaldo, 355 , \\ bairro Cerqueira César, CEP 01246-902. São Paulo, SP, Brasil. \\ E-mail: mc.barbara1029@gmail.com
}

\begin{abstract}
Thioglycolic acid is an active used in capilar cosmetic creams with the function of smoothing, curling and depilating. Resolution RDC 7/2015, from Anvisa, classifies the products in risk grade I and II. For grade I type the normative does not require detailed information about formulation, such as to the mode and restriction of use. However, grade II type needs specific indications, and their characteristics require proof of safety and/or efficacy. Norm RDC $3 / 2012$ approves the list of substances allowed in cosmetics and establish the maximum limit allowed. The products containing thioglycolic acid are classified as risk II and their content is allowed at the maximum concentration of $8.0 \% \mathrm{~W} / \mathrm{w}$ for general use, $11.0 \%$ for professional and $5 \% \mathrm{w} / \mathrm{w}$ for depilatories. The Brazilian cosmetics industry has registered a growing increase in the use of capilar straighteners, so the control of this active is important to ensure the safety of its use. The objective of this study was to optimize and validate the method for determination of the thioglycolic acid content in substitution to that published in the Guide of Quality Control of Cosmetic Products from Anvisa. The preconized methods were compared with the validation of the method under study. The method was validated using fortified sample at three concentrations $(4.0 \%, 7.0 \%$ and $10.0 \%)$. For evaluation of the results the statistical tools were the $\mathrm{T}$ and $\mathrm{F}$ tests for the comparison and validation of the method under study. The obtained results showed that the validated method is suitable for the determination of thioglycolic acid content.
\end{abstract}

Keywords: validation; titration; hair straightening; thioglycolic acid

\section{RESUMO}

O ácido tioglicólico é um ativo utilizado em cremes cosméticos capilares com a função de alisar, ondular e depilar. A Resolução RDC 7/2015 da Anvisa, classifica os produtos em grau de risco I e II. Os de grau I não requerem informações detalhadas quanto ao modo e restrição de uso e os de grau II necessitam indicações específicas e suas características exigem comprovação de segurança e/ou eficácia. A RDC 3/2012 aprova a lista de substâncias permitidas em cosméticos e estabelece os limites máximos permitidos. Os produtos contendo ácido tioglicólico são classificados como risco II e seu teor é permitido na concentração máxima de $8,0 \%(\mathrm{p} / \mathrm{p})$ para uso geral, $11,0 \% \mathrm{p} / \mathrm{p})$ profissional e $5 \%(\mathrm{p} / \mathrm{p})$ em depilatórios. O setor de cosméticos registrou um crescente aumento no uso de alisantes, sendo o controle do ativo importante para garantir a segurança de seu uso. O objetivo deste estudo foi otimizar e validar o método para determinação do teor de ácido tioglicólico em alternativa ao publicado no Guia de Controle de Qualidade de Produtos Cosméticos - Anvisa. Os métodos foram comparados e foi feita a validação do método em estudo. O método foi validado utilizando amostra fortificada em três concentrações $(4,0 \%, 7,0 \%$ e 10,0\%). Para a avaliação dos resultados foram empregadas as ferramentas estatísticas utilizando os testes t-Student e $\mathrm{F}$ para a comparação e a validação do método em estudo. Os resultados obtidos dmostraram que o método validado é adequado para determinação do teor de ácido tioglicólico.

Palavras-chave: validação; titulometria; alisantes; ácido tioglicólico. 


\section{INTRODUÇÃO}

O ácido tioglicólico é um $\alpha$-tioácido amplamente empregado na Cosmética, utilizado para reduzir rugas de expressão. Além da propriedade umectante é também empregado no tratamento dos cabelos. O uso inicial do ácido tioglicólico na Cosmética ocorreu como um depilatório, aplicação que permanece até os dias de hoje, e é responsável pelo odor desagradável de alguns cremes depilatórios (1). Esses produtos atuam pelo enfraquecimento do pelo, tornando mais fácil sua remoção, e são frequentemente encontrados na forma de loções, cremes e géis. $\mathrm{O}$ ácido tioglicólico age quebrando a ponte de dissulfeto (S-S) da queratina, a proteína que mantém o cabelo estruturado. Ao quebrar a ponte S-S, resultante do aminoácido cisteína, o pelo é mais facilmente removido do folículo. Outra aplicação é como ingrediente ativo para fazer cachos nos cabelos, conhecidos como permanente, e sua ação é novamente como um agente redutor atuando nas pontes S-S- dos cabelos. Neste caso, o cabelo é inicialmente moldado em rolos, o produto é aplicado e em seguida lavado com água a fim de parar a reação (2).

No processo de alisamento utilizando o ácido tioglicólico, a queratina é esticada. Os compostos tioglicólicos, tal como tioglicolato de amônio, são menos eficazes que o ácido tioglicólico, no entanto são mais adequados para alisamentos em cabelo lesionado por tratamentos químicos anteriores e apresentam odor mais agradável (3).

A Resolução da Diretoria Colegiada (RDC) № 7 , de 10 de fevereiro de 2015 (Anvisa), que dispõe sobre os requisitos técnicos para a regularização de produtos de higiene pessoal, cosméticos e perfumes, define e classifica os produtos cosméticos em grau I e II. Os produtos cosmético de grau I são produtos de higiene pessoal, cosméticos e perfumes cuja formulação possuí propriedades básicas ou elementar e sua comprovação não seja inicialmente necessária e não requeiram informações detalhadas quanto ao seu modo e restrição (4). Os produtos classificados como grau II apresentam formulação com indicações específicas, cujas características exigem comprovação de segurança e/ou eficácia, bem como informações e cuidados, modo e restrições de uso.

A Resolução RDC nº 3/2012 da Anvisa (5) estabelece a lista de substâncias permitidas e o limite máximo para cada ativo em suas formulações. O ácido tioglicólico apresenta o máximo de ativo de $8 \%(\mathrm{p} / \mathrm{p})$ para produtos classificados como uso geral; máximo de $11 \%(\mathrm{p} / \mathrm{p})$ e valor de $\mathrm{pH}$ entre 7,0 e 9,5 para uso profissional com a finalidade de alisar cabelos; máximo de $5 \%$ para cremes depilatórios; e máximo de $2 \%$ para outros produtos com a finalidade de tratar os cabelos (5).

Para a garantia e segurança do produto acabado é necessário verificar a concentração do princípio ativo, sendo assim importante estabelecer uma metodologia que garanta e assegure a confiabilidade dos resultados analíticos $(6,7)$. Diante do exposto, o presente estudo teve como objetivo comparar o método em estudo com aquele publicado no Guia de Controle de Qualidade de Produtos cosméticos da Agência Nacional de Vigilância Sanitária - Anvisa $(8,9)$, otimizar e validar o método para determinação do ácido tioglicólico em cremes cosméticos substituindo a solução reagente titulante de iodo, como consta no Guia da Anvisa por solução titulante de iodato de potássio. A modificação do método deve-se ao fato da solução de iodato de potássio de potássio ser mais estável e sua padronização demandar um menor tempo no preparo. O processo de validação foi realizado por meio da avaliação de parâmetros como precisão (repetitividade), precisão intermediária e exatidão (recuperação). Os limites de quantificação e detecção não foram realizados, pois já estão estabelecidos em legislação vigente e sua concentração é superior a $1 \%$ $(6,10)$. A seletividade não foi realizada pois o método foi otimizado a partir de um método normalizado. A ferramenta estatística aplicada para a avaliação dos dados obtidos foram os testes t-Student e F. Para a precisão intermediária foi aplicada Anova fator único com repetição.

\section{MATERIAL E MÉTODO}

Equipamentos: balança analítica Metller Toledo ${ }^{\circledR}$ modelo AL 204 e bureta de vidro de $25 \mathrm{~mL}$ calibrada $\mathrm{n}^{\circ}$ TEC F-86 - RBC (Rede Brasileira de Calibração).

Sais e reagentes: Ácido tioglicólico Bruno Bock Chen 99,3\%, Tiossulfato de Sódio Labsybth, iodo Synth, iodato de potássio Dinâmica ${ }^{\circledR}$, ácido clorídrico Vetec $₫$.

Amostras: para a otimização e validação da metodologia foi utilizada uma amostra branca (matriz sem analito) do creme fornecida por um fabricante de produtos cosméticos. A amostra branca foi fortificada para a obtenção de cremes alisantes em três concentrações de ativo (4,0\%, 7,0\% e 10,0\%),por adição do padrão.

A metodologia utilizada para a determinação do ativo ácido tioglicólico, cuja nomenclatura International Union of Pure and Applied Chemistry (IUPAC) (11) é 
2-sulfanylacetic acid, baseia-se na reação de oxidação do ácido tioglicólico pelo iodo em meio ácido, método publicado no Guia de Controle de Qualidade de Produtos Cosméticos - ANVISA $(8,12)$, segundo a reação:

$$
2 \mathrm{HOOC}-\left(\mathrm{CH}_{2}\right)_{\mathrm{n}}-\mathrm{SH}+\mathrm{I}_{2} \mathrm{HOOC}-\left(\mathrm{CH}_{2}\right)_{\mathrm{n}}-\mathrm{S}_{2}-\left(\mathrm{CH}_{2}\right)_{n}-\mathrm{COOH}+2 \mathrm{HI}
$$

Para o método normalizado uma quantidade de amostra contendo aproximadamente de 100 a $200 \mathrm{mg}$ de ácido tioglicólico foi pesada para cada concentração e amostra branca e em seguida foi adicionada água destilada sob agitação até total dissolução. A amostra foi acidificada com ácido clorídrico $0,1 \mathrm{M}$ e titulada com solução padronizada de iodo $0,05 \mathrm{M}$, utilizando $1 \mathrm{~mL}$ de solução de amido $1 \%$ como indicador, até a obtenção da coloração castanho-clara.

Para o método proposto, a amostra, contendo cerca de 100 a $105 \mathrm{mg}$ de ácido tioglicólico foi pesada para cada concentração e amostra branca, e acidificada com 5 $\mathrm{mL}$ de ácido clorídrico $10 \%$, dissolvendo a amostra sob agitação. Foram adicionados $0,3 \mathrm{~g}$ de iodeto de potássio e $2 \mathrm{~mL}$ de solução de amido $1 \%$ como indicador. A titulação procedeu com solução de iodato de potássio 0,02 $\mathrm{M}$, até persistir a cor violeta. $\mathrm{O}$ teor de ativo foi determinado nas três concentrações, nos dois métodos, utilizando seis replicatas. Para a validação, foram verificados os seguintes parâmetros: precisão, exatidão (recuperação) e análise estatística, que utilizou os testes t-Student e F para a comparação dos métodos. A precisão do método, foi avaliada por meio da repetibilidade onde a determinação do ativo foi realizada em seis replicatas para cada concentração. A determinação da exatidão foi realizada por ensaio de recuperação, que foi estimada pela análise de amostras fortificadas com quantidades conhecidas do analito $(4,0 \%, 7,0 \%$ e $10,0 \%)$, o que corresponde às faixas utilizadas em produtos cosméticos.

O critério para a aceitação da recuperação foi baseado na tabela da Association of Official Analytical Chemists (AOAC) (13), respeitando os níveis de concentração estimados em $98 \%$ a $102 \%$. Foram determinados a média, o desvio padrão (DP) e o desvio padrão relativo (DPR). A precisão intermediária foi determinada a partir de uma amostra de creme cosmético a base de ácido tioglicólico adquirida no comércio. $\mathrm{O}$ ensaio compreendeu a execução dos dois métodos por três diferentes analistas com seis determinações. Para a avaliação da conformidade do valor encontrado, foi solicitado à Anvisa, a disponibilização dos dados referentes ao registro e à formulação do produto. Para a comparação da metodologia foi aplicado o teste F para avaliar se as variâncias são estatisticamente iguais e em seguida foi utilizaado o teste $t$-Student para verificar se as médias dos resultados dos dois métodos podem ser consideradas estatisticamente iguais.

\section{RESULTADOS E DISCUSSÃO}

Os resultados obtidos na comparação das metodologias estão descritos na Tabela 1. Para os dois métodos a repetibilidade foi realizada em seis replicatas, cujos desvios padrão e variâncias apresentaram valores menores para o método de iodato de potássio, com exceção daqueles obtidos na concentração 10\%. Utilizou-se o teste $\mathrm{F}$ para verificar diferenças entre as variâncias dos dois métodos e o teste t-Student para comparação dos valores médios. Nas três concentrações o $F_{\text {calculado }}$ foi menor que $\mathrm{F}_{\text {crítico }}$ e p-valor maior que 0,05 , concluiu-se que não foi observado diferença significativa para as variâncias nos dois métodos. $\mathrm{O}$ valor de $\mathrm{t}_{\text {critico }}$ para 5 graus de liberdade ao nível de confiança de $95 \%$ é de 2,57 , conforme tabela 1 as concentrações de $(4,0 \%$ e $7,0 \%)$ não houve diferença significativa para os valores médios dos métodos, $\mathrm{t}_{\text {calculado }}$ menor que $\mathrm{t}_{\text {crítico }} \mathrm{e}$-valor maior que 0,05 . No entanto para a concentração de $(10,0 \%)$ assume-se que existe diferença significativa p-valor menor que 0,05 , resultado este atribuído ao teor de ativo em maior concentração. Pelo teste de Grubbs a 99\% de confiança observou-se que não houve valores dispersos nos dois métodos nas diferentes concentrações (Tabela 1).

A Tabela 2 apresenta o resultado da recuperação nos dois métodos e nas três concentrações. Em todos os casos o critério de aceitação estabelecido pelo laboratório de $(98 \%$ a $102 \%)$ foi atendido.

A precisão intermediária foi realizada utilizando uma amostra de creme alisante a base de ácido tioglicólico adquirida no comércio. $\mathrm{O}$ ensaio foi feito com o método em estudo no laboratório utilizando seis replicatas e três diferentes analistas. Para a avaliação da conformidade do valor encontrado foi solicitado à Anvisa os dados referentes ao registro e à formulação do produto, teor de ácido tioglicólico compreendido entre 4,00 e 5,00\% p/p. Os resultados estão mostrados na Tabela 3 e foram avaliados utilizando Anova fator único com repetição $F_{\text {calculado }}(1,68) F_{\text {crítico }}(3,68)$ e p-valor $(0,217)$. Conclui-se que não existe diferença significativa entre os analistas. A incerteza do ensaio foi calculada considerando fontes para o ensaio titulométrico de determinação do teor de ácido tioglicólico para um nível de confiança de 95\%. Estimou-se uma incerteza expandida de 0,03 (14). 


\section{loworma}

Tabela 1. Resultados de análise por titulação com solução de iodo e iodato das amostras de cremes cosméticos contendo ácido glicólico nas concentrações $(4,0 \%, 7,0 \%$ e $10,0 \%)$.

\begin{tabular}{|c|c|c|c|c|c|c|}
\hline Replicata & $\begin{array}{l}\text { Método (Iodo) } \\
(4,0 \%)\end{array}$ & $\begin{array}{c}\text { Método (Iodato) } \\
(4,0 \%)\end{array}$ & $\begin{array}{c}\text { Método (Iodo) } \\
(7,0 \%)\end{array}$ & $\begin{array}{l}\text { Método (Iodato) } \\
(7,0 \%)\end{array}$ & $\begin{array}{c}\text { Método (Iodo) } \\
(10,0 \%)\end{array}$ & $\begin{array}{c}\text { Método (Iodato) } \\
(10,0 \%)\end{array}$ \\
\hline 1 & 3,99 & 4,02 & 7,09 & 7,13 & 9,98 & 10,12 \\
\hline 2 & 3,83 & 3,97 & 7,06 & 7,03 & 9,96 & 10,18 \\
\hline 3 & 3,89 & 4,03 & 6,80 & 7,00 & 9,99 & 10,11 \\
\hline 4 & 3,87 & 4,07 & 7,05 & 6,99 & 9,94 & 10,15 \\
\hline 5 & 3,99 & 3,99 & 6,95 & 7,06 & 9,98 & 10,18 \\
\hline 6 & 3,98 & 3,98 & 7,07 & 7,05 & 9,97 & 10,16 \\
\hline Média & 3,92 & 4,01 & 7,00 & 7,04 & 9,97 & 10,15 \\
\hline$D P^{*}$ & 0,07 & 0,04 & 0,11 & 0,05 & 0,02 & 0,03 \\
\hline $\mathrm{DPR}^{* *}$ & 1,79 & 0,93 & 1,58 & 0,72 & 0,18 & 0,30 \\
\hline Variância & 0,005 & 0,001 & 0,0123 & 0,003 & 0,0003 & 0,001 \\
\hline Recuperação & 98,12 & 100,25 & 100,05 & 100,62 & 99,70 & 101,50 \\
\hline $\begin{array}{c}\mathrm{G}_{\text {máximo calc }} \\
\mathrm{G}_{\text {crítico }}\end{array}$ & $\begin{array}{l}0,924 \\
1,350 \\
1,973\end{array}$ & $\begin{array}{l}1,604 \\
1,069 \\
1,973\end{array}$ & $\begin{array}{l}0,781 \\
1,833 \\
1,973\end{array}$ & $\begin{array}{l}1,717 \\
1,057 \\
1,793\end{array}$ & $\begin{array}{l}1,118 \\
1,677 \\
1,973\end{array}$ & $\begin{array}{l}1,011 \\
1,348 \\
1,973\end{array}$ \\
\hline $\begin{array}{c}\mathrm{F}_{\text {calculado }} \\
\mathrm{F}_{\text {crítico }} \\
\text { valor - p }\end{array}$ & $\begin{array}{l}3,536 \\
7,146 \\
0,192\end{array}$ & & $\begin{array}{l}4,832 \\
7,146 \\
0,109\end{array}$ & & $\begin{array}{l}2,750 \\
7,146 \\
0,291\end{array}$ & \\
\hline $\begin{array}{c}T_{\text {calculado }} \\
T^{\text {critico }} \\
\text { valor - } p\end{array}$ & $\begin{array}{l}-2,429 \\
2,571 \\
0,06\end{array}$ & & $\begin{array}{l}-0,990 \\
2,571 \\
0,368\end{array}$ & & $\begin{array}{l}-10,887 \\
2,571 \\
0,000\end{array}$ & \\
\hline
\end{tabular}

${ }^{*} D P=$ Desvio padrão **DPR $=$ Desvio padrão relativo.

Tabela 2. Resultado da recuperação, média, desvio padrão, desvio padrão relativo e variância do método de iodo e iodato de potássio obtidos da análise das amostras de produtos contendo ácido tioglicólico nas concentrações de 4,0\%, 7,0\% e 10,0\%.

\begin{tabular}{|ccccccc}
$\begin{array}{c}\text { Concentração teórica (\%) } \mathbf{p} / \mathbf{p} \\
\text { Titulação iodo / iodato }\end{array}$ & Recuperação (\%) & Média & DP* & DPR* * & Variância \\
\hline Iodo 4,0 & 98,13 & 3,92 & 0,070 & 1,78 & 0,005 \\
Iodo 7,0 & 100,05 & 7,00 & 0,100 & 1,43 & 0,010 \\
Iodo 10,0 & 99,70 & 9,97 & 0,018 & 0,18 & 0,000 \\
Iodato 4,0 & 100,25 & 4,01 & 0,040 & 1,00 & 0,002 \\
Iodato 7,0 & 100,62 & 7,04 & 0,050 & 0,71 & 0,002 \\
Iodato 10,0 & 101,50 & 10,15 & 0,030 & 0,29 & 0,001
\end{tabular}

${ }^{*} D P=$ Desvio padrão ${ }^{*} D P R=$ Desvio padrão relativo.

A Tabela 2 apresenta o resultado da recuperação nos dois métodos e nas três concentrações. Em todos os casos o critério de aceitação estabelecido pelo laboratório de $(98 \%$ a $102 \%)$ foi atendido.

A precisão intermediária foi avaliada utilizando uma amostra de creme alisante com ácido tioglicólico, adquirido no comércio. O ensaio foi feito com o método em estudo no laboratório, utilizando seis replicatas e três diferentes analistas. Para a avaliação da conformidade do valor encontrado, foi solicitado à Anvisa os dados referentes ao registro e à formulação do produto, teor de ácido tioglicólico compreendido entre 4,00 e 5,00\% p/p. 
Os resultados estão demonstrados na Tabela 3 e foram avaliados utilizando Anova fator único com repetição $F_{\text {calculado }}(1,68) F_{\text {critico }}(3,68)$ e p-valor $(0,217)$. Conclui-se que não existe diferença significativa entre os analistas. A incerteza do ensaio foi calculada considerando fontes para o ensaio titulométrico de determinação do teor de ácido tioglicólico para um nível de confiança de 95\%. Estimou-se uma incerteza expandida de 0,03 (14).

\section{CONCLUSÃO}

A otimização e validação da metodologia de determinação de ácido tioglicólico por titulometria utilizando o iodato de potássio como titulante, mostrou-se uma técnica precisa, rápida, de baixo consumo de reagentes e assegura a confiabilidade dos resultados

Tabela 3. Resultados do teor de ácido tioglicólico para avaliar a precisão intermediária de uma amostra de creme cosmético obtida no comércio com três analistas.

\begin{tabular}{|cccc|}
\hline Determinaçöes $(\mathbf{N})$ & Analista $\mathbf{1}$ & Analista 2 & Analista 3 \\
\hline 1 & 4,60 & 4,70 & 4,61 \\
\hline 2 & 4,62 & 4,68 & 4,71 \\
\hline 3 & 4,70 & 4,85 & 4,63 \\
\hline 4 & 4,68 & 4,72 & 4,80 \\
\hline 5 & 4,63 & 4,68 & 4,61 \\
\hline 6 & 4,74 & 4,70 & 4,58 \\
\hline Média & 4,66 & 4,72 & 4,66 \\
\hline DP* & 0,05 & 0,06 & 0,08 \\
\hline DPR* & 1,15 & 1,37 & 1,78 \\
\hline
\end{tabular}

* $D P=$ Desvio padrão ${ }^{*} D P R=$ Desvio padrão relativo.

\section{REFERÊNCIAS}

1. Sousa KS, Augusto Filha VLS, Hugo V, Pinto A, Fonseca MG, Espínola JGP, Arakaki LNH. Quimissorção de cátions divalentes em sílica gel modificada com ácido tioglicólico - a influência do $\mathrm{pH}$ e força iônica. Quím. Nova 2007; 30 (3): 528-534. DOI 10.1590/S010040422007000300005.

2. Souza AMF, Santana DP, Silva JA, Lima ZN. Avaliação da qualidade de alisantes capilares: determinação da segurança de ingredientes ativos e contaminantes microbiológicos. Rev. Bras. Farm. 2012; 93(3):331-336.

3. Wilkinson JB, Moore RJ. Cosmetologia de Harry. Madrid. Ediciones Diaz de Santos; 1990.

4. BRASIL. Ministério da Saúde. Agência Nacional de Vigilância Sanitária. Resolução RDC nº 07 de 10 de fevereiro de 2015. Dispõe sobre os requisitos técnicos para a regularização de produtos de higiene pessoal, cosméticos e perfumes. Diário Oficial [da] República Federativa do Brasil, Poder Executivo, Brasília, DF, 11 fev.2015.

5. BRASIL. Ministério da Saúde. Agência Nacional de Vigilância Sanitária. Resolução RDC n ${ }^{\circ} 03$ de 18 de janeiro de 2012. Aprova o Regulamento Técnico Listas de
Substâncias que os produtos de Higiene Pessoal, cosméticos e Perfumes não devem conter exceto nas condições e com restrições estabelecidas. Diário Oficial [da] República Federativa do Brasil, Poder Executivo, Brasília, DF, 20 jan.2015.

6. ABNT. Associação Brasileira de Normas Técnicas. NBR ISO/IEC 17025. Requisitos gerais para a competência de laboratórios de ensaio e calibração. Rio de Janeiro: ABNT, 2005.

7. Polonini HC, Santos FC, Vaz UP, Brandão MAF. Desenvolvimento e validação de método analítico para determinação do teor de sinvastatina em cápsulas magistrais. Quím. Nova. 2011; 34 (3): 516-519. DOI 10.1590/ S0100-40422011000300026.

8. ANVISA. Guia de controle de qualidade de produtos cosméticos. Uma abordagem sobre os ensaios físicos e químicos. Brasília: Anvisa. 2008. 120p.

9. Alves AI, Teixeira LJQ, Saraiva SH, Carneiro JS. Revalidação do método titulométrico para determinação do ácido ascórbico. Rev Ceres Viçosa. 2014; 61(2):155-161. DOI 10.1590/S0034-737X2014000200001 


\section{Inotarma}

10. INMETRO. Instituto Nacional de Metrologia, Qualidade e Tecnologia. DOQ-CGCRE-008: Orientação sobre validação de métodos analíticos. Rio de Janeiro: INMETRO; 2016. 31p.

11. Rodrigues JA. Nomenclatura de compostos orgânicos segundo as recomendações da IUPAC. Uma breve introdução. Chemkeys: Liberdade para aprender, jul. 2011. Disponível em: <http://chemkeys.com/br/2011/07/15/ nomenclatura-de-compostos-organicos-segundo-as-recomendacoes-da-iupac-uma-breve-introducao $>$. Acesso em: 24/05/2017.
12. Souza AMF, Santana DP, Silva JA, Lima ZN. Avaliação da qualidade de alisantes: determinação da segurança quanto à análise de ingredientes ativos e contaminantes microbiológicos. Rev. Bras. Farm. 2012; 93(3): 331-336.

13. AOAC. Association of Official Analytical Chemists. Official methods of analysis. Appendix D: guidelines for collaborative study procedures to validate characteristics of a method of analysis. Washington: AOAC, 2002.

14. EURACHEM/CITAC Guide. Measurement uncertainty arising from sampling: a guide to methods and approaches Eurachem. 1st ed. 2007. 